\section{Tuberculous meningitis in adults: a review of a decade of developments focusing on prognostic factors for outcome}

\author{
Flavia Brancusi' , Jeremy Farrar ${ }^{2}$ \& Dorothee Heemskerk ${ }^{\star 2}$ \\ 'Princeton University, Princeton, NJ, USA \\ ${ }^{2}$ Oxford University Clinical Research Unit, Ho Chi Minh City, Vietnam \\ *Author for correspondence: dheemskerk@oucru.org
}

Tuberculous meningitis (TBM) is the most severe form of TB. Despite treatment, mortality and long-term disability remain unacceptably high. Prevention, early recognition, diagnosis and treatment are fundamental to improving outcomes. However, an effective vaccine remains elusive, initial symptoms are nonspecific, and sensitive diagnostic tests are not available. There has been progress in our understanding of the immunopathology of TBM, and several factors have been found to be associated with susceptibility to infection, disease progression and clinical outcome. However, these have not yet impacted on treatment. Early treatment initiation and uninterrupted continuation, severity on presentation, seizures, stroke, cranial nerve involvement, cerebrospinal fluid cell count and lactate levels, hyponatreamia and coinfection with HIV are all found to be important prognostic factors for outcome. Pathogen lineage (Beijing genotype) and host genetics (polymorphisms in TLR2, TIRAP and LTA4H genes) can influence susceptibility to TBM. However, these findings have not yet impacted on treatment. Progress in vaccine development, opportunities for better diagnostic tests, novel insights into pathogenesis and an increasing evidence base for improving treatment should impact the current high mortality and morbidity, if translated to global and local guidelines.

\section{Burden of disease}

TB is second to HIV as an infectious cause of death globally. In 2009, the global prevalence was 14 million, incidence was 9.4 million and mortality due to TB was 1.7 million [1]. An estimated 2 billion people carry latent infection, which represents a vast reservoir for future active TB cases. The incidence of extrapulmonary $\mathrm{TB}$ is directly related to the prevalence of TB infection. CNS TB comprises approximately $10 \%$ of all $\mathrm{TB}$ cases [2].

Of the extrapulmonary forms of TB, tuberculous meningitis (TBM) is the most severe. It is difficult to diagnose and treat for a number of reasons: the pathology is poorly understood; rapid, sensitive and affordable diagnostics have yet to be developed; drug resistance is crucial to clinical outcome, and the optimal dose, duration and composition of anti-TB treatment regimens have still not been determined [3]. The HIV epidemic has complicated all aspects of TB management. Infection is more likely to progress to meningeal TB when patients are coinfected with HIV. In HIV-negative adults, the mortality is approximately $25 \%$; for HIVpositive patients it is $67 \%[4,5]$. Approximately half of survivors suffer from long-term neurological sequelae $[4,6]$. Neurological disability frequently involves cognitive impairment, motor deficits, optic atrophy and other cranial nerve involvement [7]. Disability may be secondary to neurological dysfunction, such as airway or urinary tract complications [8]. In a long-term outcome follow-up study, there was no benefit of dexamethasone on disability or survival after 5 years; overall, approximately $26 \%$ had good outcome, $16 \%$ had intermediate disability, 7\% were severely disabled and 51\% had died [9].

Severity on presentation is prognostic for outcome. The British Medical Research Council published a set of clinical criteria for assessing the severity of TBM as follows: fully conscious, nonspecific symptoms (stage I); lethargy or cranial nerve palsies (stage II); stupor, severe illness, gross paralysis or paresis (stage III) [10]. For HIV-negative patients presenting with stage I disease, mortality is approximately $20 \%$; for stage II: $30 \%$; and for stage III: $55 \%$ [4]. In patients coinfected with HIV, these figures may be as high as 40, 52 and $75 \%$, respectively, if antiretroviral therapy (ART) is initiated in the continuation phase of TB treatment [5].

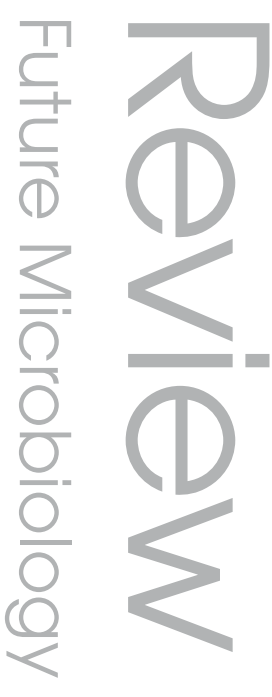

\section{Keywords \\ = adult $=$ diagnosis $\approx$ genetics - immunopathology \\ - prognostic factor = review - treatment = tuberculosis \\ - tuberculous meningitis}

\section{Future
Medicine $\%$ fart of}




\section{Immunopathogenesis}

Pathology

Following inhalation of airborne droplets containing Mycobacterium tuberculosis (Mtb), the ingestion of $\mathrm{Mtb}$ by alveolar macrophages triggers an inflammatory cascade, generating protective immunity and formation of the primary complex. During a short period of bacteremia, bacilli can hematogeneously seed elsewhere in the body, including the meninges [11-14]. Isolated granuloma can form throughout the surface of the brain, known as Rich foci. Brain pathology may arise from an intense inflammatory reaction to bacilli and tuberculous antigens released from lesions into the subarachnoid space. Exudate may form and cause adhesions blocking the flow and reabsorption of the cerebrospinal fluid (CSF). Pressure may build up, leading to hydrocephalus, ischemia and infarction $[3,8,12,14-16]$. The original pathological studies on which this description is based were performed in 1933 by Rich and McCordock [12]. Their work has been quoted in the majority of texts on TBM for over 80 years.

A current review has elaborated on the ancient pathological studies, incorporating more recent pathological, post-mortem, animal and epidemiological data. Whereas Rich and McCordock denied a causal relationship between the frequently co-existing miliary TB and TBM, the authors of the current review are of the opinion that a pathogenetic relationship is very likely. Based on recent pathological findings, they propose a classification of pathogenesis in several groups, in which the Rich focus remains central, but including miliary $\mathrm{TB}$ as an exacerbated form of hematogenous spread, having an important role in establishing the cortical and meningeal focus [11]. This critical review explicates the frequently found occurrence of miliary TB in TBM patients (Figure 1). Human autopsy studies in TBM remain rare, but are essential to deepen our understanding of pathogenesis.

Discussion of other form of CNS TB, such as tuberculoma, abcesses, cerebritis, encephalitis, spinal and military TB without a clinical picture of meningitis fall outside the scope of this article.

\section{Imaging}

MRI is the modality of choice for detecting abnormalities in TBM, but computed tomography (CT) is also commonly used [17-19]. Newer sequences of MRI, magnetic resonance spectroscopy and altered dosing of gadolinium have been proposed to increase sensitivity, but currently are still of questionable value since specificity remains an issue and most settings have no access to these techniques.

Imaging studies have shown that hydrocephalus, tuberculoma and meningeal enhancement (FIgUre 2) are common features at diagnosis of TBM [20-22]. Tuberculoma typically presents as a ringenhancing lesion, solitary, but more commonly multiple, and may vary significantly in shape and size [20]. They have been reported to be present on initial MRI in up to $39 \%$ of patients with TBM, but paradoxically may develop during antitubercular treatment [23]. The presence of tuberculoma is not prognostic for outcome, but tuberculoma in the frontal, temporal and optochiasmatic regions tend to carry a worse prognosis than tuberculoma elsewhere in the brain [23]. Cranial nerve involvement is common in TBM and occurs in 20-30\% of patients [2]. Optochiasmatic arachnoiditis and hydrocephalus on MRI are predictors of the presence of cranial neuropathy, which is associated with poor outcome [24].

HIV-infected adults had similar MRI patterns as HIV-negative patients, although the differential diagnosis may include other opportunistic infections [25].

Patients rarely present with infarction, but within 12 months following presentation it occurs in up to $60 \%$ of patients. Most commonly affected are the basal ganglia, predominantly the anterior part, whereas ischemic stroke occurs more frequently in the posterior region [19,20,25,26-28]. Infarction may be asymptomatic, but can also cause severe disability and death, and is associated with poor outcome [19,29]. Predictors of stroke are advanced stage of TBM, basal exudates, optochiasmatic arachnoiditis and vision impairment [30]. In the randomized controlled trial on dexamethasone, the proportion of patients with infarction was halved in a serial MRI study, but this was not statistically significant [19]. The ability to predict and prevent stroke by neuroimaging may have a major impact on morbidity and mortality. MR angiography (MRA) may have a role in predicting the chance of infarction in TBM, as recently an Indian group has found more than $40 \%$ of patients with abnormal MRA went on to develop infarcts, but no infarcts occurred in the normal MRA group [26].

\section{Immunology}

The immune response within the brain in TBM remains poorly understood. Morbidity and mortality are considered to be dependent on the severity of the inflammatory response, and this 
provided the rationale for using adjunctive treatment with dexamethasone. However, immunological studies provide conflicting results, creating a paradox between the hypotheses and the evidence, further complicated by the association of TBM with HIV. Typically in TBM, the CSF shows a lymphocytic pleiocytosis and inflammatory indices (including TNF, IFN- $\gamma$, IL-8, IL-10, matrix metalloproteinases, and their corresponding tissue inhibitors) are usually raised in the CSF in the acute phase of disease. The blood-brain barrier is often damaged, reflected by a raised CSF:serum albumin ratio [31-33]. In HIV-negative patients, the concentration of lactate and protein are positively associated with TBM grade and clinical outcome [31]. Lactate is produced in hypoxic tissues, and high levels in CSF may reflect ischemic events in the brain. IL-8, IFN- $\gamma$, TNF- $\alpha$, MMP9 and TIMP1 levels in CSF have been shown to correlate with lactate levels, suggesting a role in pathogenesis of infarction, but were not correlated with outcome. Only IL-6 showed an independent association with stage III disease in HIV-negative patients [8]. The exact role in pathogenesis of these cytokines remains to be established, and the CSF may not reflect the response within neuronal tissue.

The cellularity of CSF plays a role in disease severity. Low white cell counts in the CSF are associated with mortality. Survival, however, was associated with a low percentage of lymphocytes and a higher proportion of neutrophils, suggesting that a neutrophilic response may be protective [8].

HIV infection is known to attenuate the immune response in CSF, and HIV-infected patients more commonly have less inflammatory CSF [34]. In Vietnam, HIV-positive individuals had an inverted CSF IFN- $\gamma$ :IL-10 ratio, which may represent a difference in the balance of pro-/anti-inflammatory cytokines. Interestingly, a low IFN- $\gamma$ was associated with death in HIV-positive patients, implicating that a proinflammatory response may actually be protective of death in TBM in that population [34].

In vitro studies have shown microglial cells (resident macrophages of the brain) produce robust amounts of cytokines and chemokines (TNF- $\alpha$, IL-6, IL-1B, CCL21, CCL5 and CXCL10) in response to infection with Mtb. In the presence of dexamethasone, the production of these proinflammatory mediators diminished [35]. Paradoxically, in clinical studies, dexamethasone did not attenuate the inflammatory indices in the CSF [36].
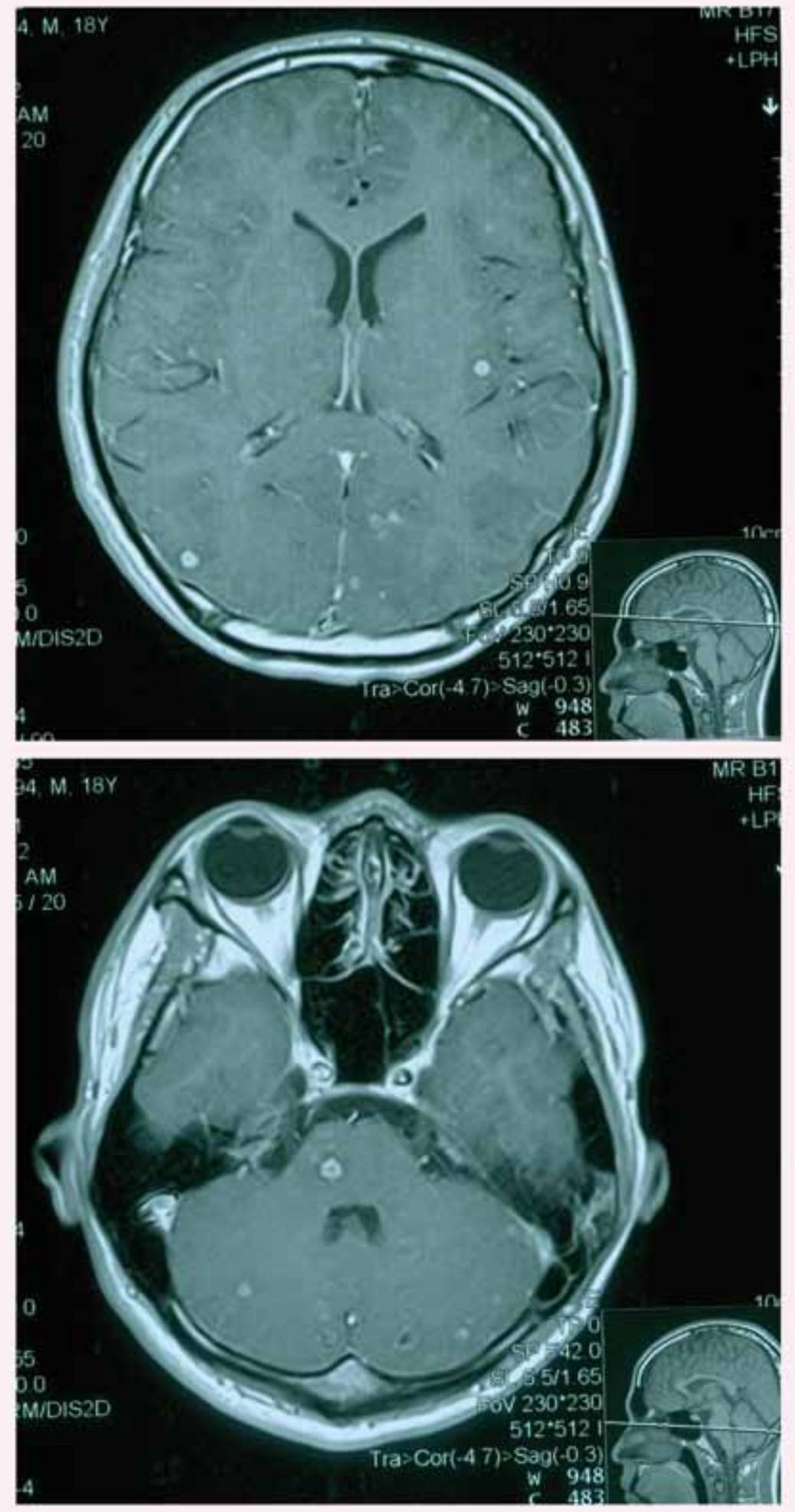

Figure 1. Miliary TB in an 18-year-old male with tuberculous meningitis.

A recent publication on host-genetic susceptibility to mycobacteria proposed a theory to explain the inflammatory response in humans [37]. The study included human genetics, a susceptibility model of zebrafish to infection by 

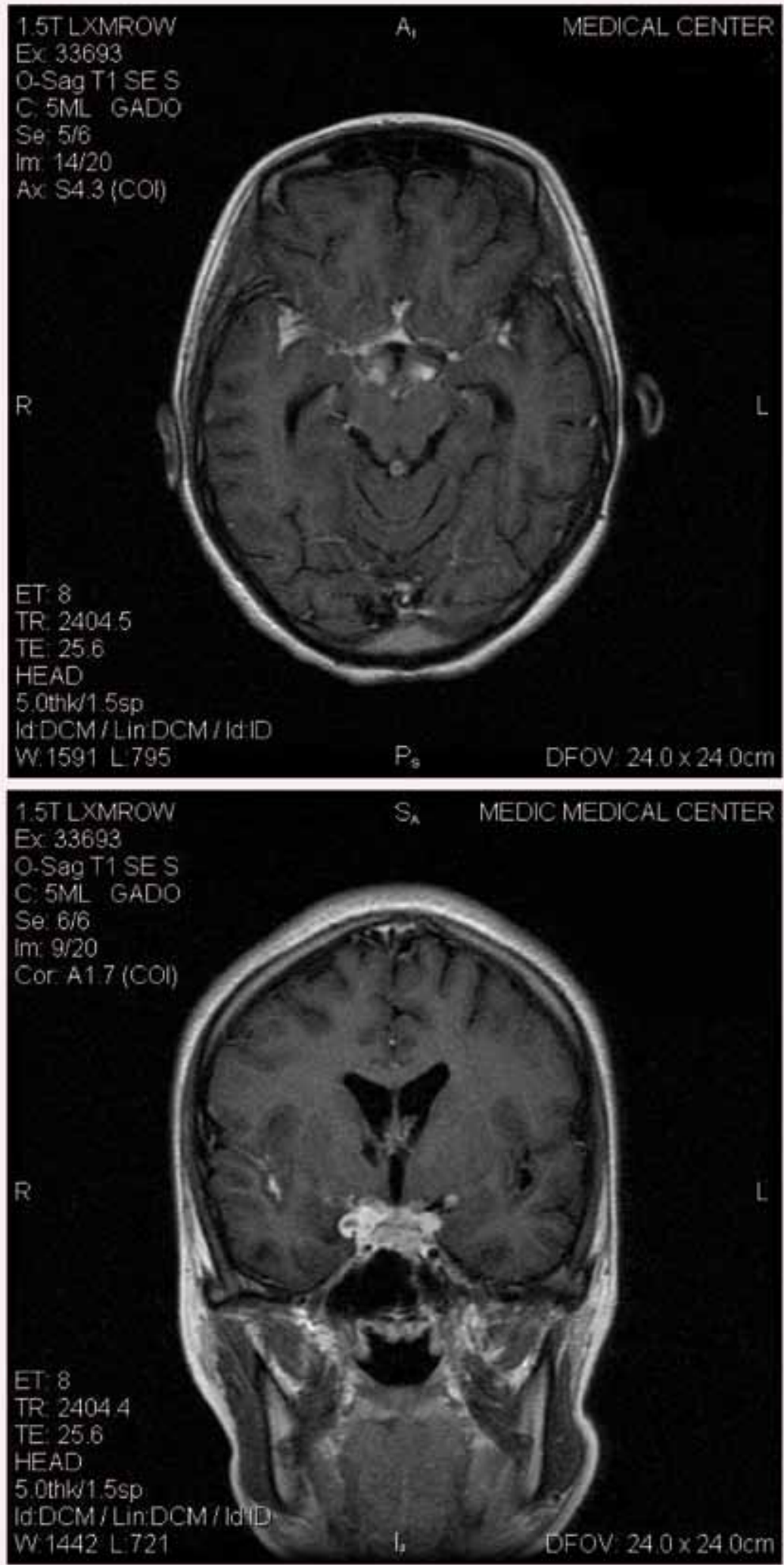

Figure 2. Basal meningeal enhancement in a 15-year-old female with tuberculous meningitis. modulated through the $L T A 4 H$ gene. Indirectly, this gene can disregulate TNF levels and cause increased susceptibility to TBM either through a hyperinflammatory response or inadequate inflammation. In humans, a polymorphism in the $L T A 4 H$ promotor region has been identified, and patients with TBM who were homozygous for the $L T A 4 H$-high polymorphism had higher levels of TNF and high leukocytes in CSF. Mortality was higher in these patients when not treated with glucocorticoids, but adjunctive treatment with dexamethasone resulted in a reduction of death. Patients who were homozygous for the polymorphism causing low expression of $L T A 4 H$ also had high mortality rates, regardless of treatment with glucocorticoids. The patients who were heterozygous at this locus had the lowest mortality [37].

These results suggest that disease severity can be caused either by an exaggerated or a deficient immune response, and perhaps more importantly, that treatment with corticosteroids may benefit only those with a certain predisposition to hyperinflammation and may be detrimental in those with deficient immune responses. Conflicting immunological data may stem from the fact that patients differ in the genetic control of their immunological response to infection with TBM.

The immunological mechanisms in response to the bacillus Calmette-Guérin (BCG) vaccine are ambiguous. BCG immunization has been shown to be effective in prevention of childhood TB, especially miliary TB and TBM [38]. Data on the effect on severity of symptoms in pediatric TBM are conflicting. The prevention of TBM in adults has not been established as the effect wanes after 5-10 years and benefit of vaccination of HIV-positive individuals is unknown [39]. The severity or prognosis of TBM in BCGvaccinated adults does not seem to differ from that of nonvaccinated patients [7].

\section{Pathogen \& host genetics Pathogen genetics}

The 4.41-Mb genome of $\mathrm{Mtb}$ has been sequenced [40]. Half of the approximately 4000 known protein-coding genes have been assigned a function, and 376 putative proteins share no homology with known proteins and are presumably unique to Mtb [15]. This information is crucial for identifying genes that code for virulence factors and antigens against which host immunity is directed.

Varying genotypes exist within four main lineages found globally: Indo-Oceanic, east Asian, east African/Indian and European/American [41]. 
Within the east Asian lineage, the Beijing genotype has been extensively investigated, as it is widespread and associated with increased virulence. It shows significant association with HIV status, single- and multi-drug resistance [42]. Patients infected with Beijing strain had shorter duration of illness before presentation to hospital and fewer CSF leukocytes, suggesting that mycobacterial genotype may affect disease progression and the intracerebral inflammatory response [43]. A recent retrospective cohort study of TBM patients in Thailand supported the notion that the Beijing genotype is the most pathogenic strain of Mtb and associated with TBM, while the Euro-American lineage was much less commonly linked with TBM. Results showed modern sublineages of Beijing genotype were associated with higher CSF white cell count and more severe disease (stage III), but not with mortality rate [44]. In HIV-positive patients in Vietnam, those infected with the modern Beijing lineage strains had lower mortality than patients infected with the ancient Indo-Oceanic lineage (hazard ratio [HR]: 0.29) [45]. This contradictory finding may be explained by the proinflammatory properties of the modern Beijing lineages that may be detrimental to the immunocompetent host, but conversely may possibly protect the immunocompromised.

\section{Host genetics}

Several candidate genes have been proposed to be associated with susceptibility for pulmonary $\mathrm{TB}$ [46-49]. Using these candidate genes, the quest for polymorphisms associated with disseminated disease has begun. The Toll-like receptor (TLR) pathway has been implicated in TB progression. The human TLR family has 12 members that can recognize pathogen-associated molecular patterns and, upon activation, initiate an innate immune response, cytokine production and the formation of the adaptive immune response. The TLRs known to be involved in Mtb recognition are TLR2, TLR4, TLR9 and possibly TLR8 [50]. One of the few host genetic studies performed on TBM patients showed an association between a polymorphism in the TLR2 gene (single nucleotide polymorphism T597C) and the development of TBM and miliary TB, indicating TLR2 influences the dissemination of Mtb [51]. A polymorphism in the TIRAP gene has also been shown to be associated with susceptibility to TBM [52]. TIRAP is a protein further down the TLR pathway, which mediates signals from the TLR receptors, activating macrophages and dendritic cells.
Recently, a single nucleotide polymorphism in the promoter region of the $L T A 4 H$ gene has been identified to play a role in susceptibility for mycobacteria, as discussed earlier. High levels of LTA4H trigger a cascade which will cause high levels of TNF (through upregulated leukotriene levels), thus hyperinflammation. Low levels of LTA4H cause low levels of TNF (through upregulated lipoxin levels) and consequently, insufficient inflammation [37].

Pathogenesis may be more heterogenous than previously thought. Vulnerability is multifactorial, involving a chain of events that may differ between individuals depending on age, genetic make-up, pathogen virulence, HIV coinfection and possibly other factors that have not yet been identified.

\section{Diagnosis}

The early clinical features of TBM are nonspecific, making the initial differential diagnosis wide. Rapid diagnosis and initiation of effective antituberculous therapy are fundamental to outcome, as demonstrated by the high mortality in patients who present late and are already in stage II or III on admission.

\section{Microbiological \& clinical diagnosis}

The gold standard for diagnosis of TBM is the demonstration of acid-fast bacilli (AFB) in the CSF, either by Ziehl-Neelsen (ZN) smear microscopy or Mtb isolated by culture. The sensitivity of smear microscopy is generally low, and depends critically on the capacity of laboratories, technician experience and diligence; $\mathrm{ZN}$ smear sensitivity can vary between 10 and $60 \%$. Culture positivity rates are between 25 and $75 \%$, but results are only available after 2-6 weeks of incubation [13]. Increasing the CSF sample volume (minimum $6 \mathrm{ml}$ ) and duration of slide examination increases the sensitivity of smear microscopy (to 60\%) [53]. Improving inhospital logistics and organization can improve the yield from CSF. Close cooperation between clinical ward and biochemistry, hematology and microbiology laboratories helps ensure that the precious CSF sample is used most efficiently, reducing to a minimum the amount of CSF used in the biochemical and hematology tests, preserving maximum volume for $\mathrm{ZN}$ stain and culture. Filtration of CSF may be a simple method to improve mycobacterial isolation and culture sensitivity [54].

Some institutions may additionally perform the microscopically observed drug sensitivity (MODS) assay and/or very recently developed 
GenXpert $^{\circledR}$ test (Cepheid, Sunnydale, CA, USA), since their benefit has been shown in terms of time-to-result compared with culture [55-57]. MODS is an inexpensive technique that can identify drug-resistant Mtb through direct drug susceptibility testing (DST) in liquid culture. For CSF, after primary isolation, samples inoculated in MODS can turn positive after a median of 6 days, significantly faster than mycobacterial growth indicator tube (MGIT; 15.5 days) and Lowenstein-Jensen culture (24 days) [55].

A definite diagnosis of TBM is made when AFB are detected in the CSF. Since ZN smear has low sensitivity and prompt treatment is crucial, in practice, diagnosis of TBM is based on a combination of clinical criteria, evidence from CSF, imaging results and isolation of AFB from other sites. The diagnosis of TBM can be 'definite', 'probable' or 'possible' depending on the solidness of the clinical evidence [58]. The criteria for 'probable' and 'possible' TBM are not uniform, complicating comparisons between studies and reproducibility of results. In 2009, a standardized clinical case definition for TBM was published [59]. Although based on expert opinion rather than a formal systematic review or 'grade' analysis, the standardized definitions of 'definite', 'probable' and 'possible' TBM should prove helpful in improving the design of clinical studies and care of patients globally.

\section{Diagnostic tests}

There is an urgent need to improve early diagnosis. In the past decade, many different diagnostic tests for TBM have been studied, yet all lack sensitivity. There are major hurdles in establishing a proven diagnosis in TBM. Central in diagnosis is the lumbar puncture. The procedure is invasive and particularly in patients in coma or with signs of raised intracranial pressure, it may not be safe. In many settings it may be impossible to perform brain imaging where TBM is common. If CT or MRI of the brain can be performed prior to lumbar puncture, it is still not clear how to make a reliable prediction who is at highest risk of coning or other complications. In many cases, TB treatment may have been started before lumbar puncture is performed, on clinical grounds; this affects the sensitivity of diagnostic tests. For nucleic acid amplification tests, contamination of samples may influence specificity. Although this review is not exhaustive on this topic, we would like to discuss some recent developments in TBM diagnostics.

\section{Adenosine deaminase}

Biochemical tests aim to detect features of Mtb or products of the host immune response. Adenosine deaminase (ADA) has been of interest for many years in TB diagnosis. ADA was first isolated from calf mucosa in the early 1940 [60]. ADA is ubiquitously present in the body, but especially in lymphoid tissue, levels are particularly high in active $\mathrm{T}$ lymphocytes, hence it is associated with disorders that induce T-cellmediated immune responses. It has been shown to be of value in the distinction of tuberculous pleural effusions [61]. The use in differentiating between TBM and other forms of meningitis is attractive, because it is a relatively inexpensive and easy test, especially important in lowincome settings. Numerous studies have been published regarding the usefulness in TBM. A recent meta-analysis concluded that the mean sensitivity and specificity of ADA assays were 79 and $91 \%$, respectively [62]. However, publication bias may have resulted in overestimation of diagnostic accuracy. Generally, ADA assays may be useful in confirming TBM, but raised levels may also be seen in other CNS disorders (sarcoidosis, meningeal lymphoma, subarachnoid hemorrhage, neurobrucellosis), rendering it too nonspecific $[13,63,64]$. It is not a useful test in HIV-positive patients [13].

\section{Lipoarabinomannan}

Various immunoassays have been evaluated for diagnosis of TBM, with highly variable sensitivity and specificity between studies. The pitfall of immunoassays is that they may not distinguish between acute infection and previous exposure, and cross-reactivity of antibodies may further influence specificity. Of the antigen assays, the detection of lipoarabinomannan (LAM) has been of recent interest. LAM is a cell wall component of Mtb, has immunoregulatory and anti-inflammatory effects, and serves as a virulence factor of mycobacteria [65]. Recently, a standardized LAM antigen detection ELISA test showing results within 2-3 h, has been developed (Clearview ${ }^{\circledR}$ TB ELISA, Inverness Medical Innovations, MA, USA). It was evaluated on CSF in a high HIV prevalence setting; the sensitivity of the LAM detection assay was $14 \%$ and specificity $94 \%$. Combined with clinical markers, the sensitivity increased to $38 \%$. The authors concluded that combining the clinical prediction rule with LAM antigen detection increased the rule-in value for TBM [66]. On its own it is inadequate to rule-out TBM, so further work would need to be done before this test adds significantly to the current 
diagnostic algorithm. We do not believe currently available immunoassays have any role in the diagnosis of TBM.

\section{GeneXpert}

Amplification of mycobacterial DNA by PCR has turned away attention from immunological techniques. A systematic review on commercial nucleic acid amplification tests (NAATs) reported a potential to rule-in or confirm diagnosis, but low sensitivity precludes the use of these tests to rule-out TBM. Overall sensitivity and specificity were reported to be 56 and $98 \%$, respectively [67]. NAAT cannot replace conventional microscopy or culture and should be used in combination with clinical algorithms and existing diagnostics $[67,68]$.

In December 2010, the WHO for the first time endorsed a new rapid diagnostic test for TB, the GeneXpert MTB/RIF (Cepheid). The GeneXpert is a desktop machine that simultaneously detects the presence of Mtb and rifampicin resistance in specimens, using nested real-time PCR. The advantages of this novel commercial NAAT are the ease of use for inexperienced staff and rapid turnover time $(\sim 2 \mathrm{~h})$. Risk of contamination and consequent false-positive results is reduced by the use of sealed disposable cartridges. Its ability to detect rifampicin resistance within $2 \mathrm{~h}$ may lead to an important improvement in the management of multidrug-resistant (MDR)-TB [69]. Limitations of the test include the current cost of the machine (approximately US $\$ 17,000)$ and the individual cartridges (\$17-20). The cartridges have a limited shelf-life and the machine needs yearly maintenance by experienced personnel. The sensitivity for pulmonary TB is reported to be higher than smear, but less than culture [70]. In smear-negative, culture-positive sputum samples, detection of Mtb showed $76.9 \%$ sensitivity and $99.0 \%$ specificity, in a decentralized low-income setting [70]. Sensitivity for rifampicin resistance was $94.4 \%$ and specificity $98.3 \%$, regardless of HIV infection [70]. These results are clearly promising and roll-out of the GeneXpert has begun in several countries. GeneXpert on extrapulmonary samples showed a combined sensitivity and specificity of 77.3 and $98.2 \%$, respectively [71]. However promising, only 19 of the 521 nonrespiratory specimens were CSF. The GeneXpert test still has to be validated for CSF samples. For TBM, this rapid molecular test may be of great importance, as delay in diagnosis is directly linked to mortality. Culture and drug sensitivity testing (DST) results will only become available after at least 6 weeks. Most patients with rifampicin resistance will have died within the first month of treatment [45,72]. Further evaluation of this novel GeneXpert assay may lead to significant improvement in the diagnosis and treatment of TBM in general and MDR-TBM in particular.

\section{Treatment \\ Specific antituberculous treatment}

Global treatment guidelines for TBM treatment are not uniform, are based on pulmonary regimes, and have not been evaluated with randomized controlled trials. Guidelines generally recommend treatment with rifampicin, isoniazid, pyrazinamide and streptomycin (or ethambutol) for 3 months in the intensive phase, followed by at least a 6 -month period of treatment with rifampicin and isoniazid [1,73,74].

Antituberculous drugs are known to have variable CSF penetration. In particular, CSF penetration of rifampicin, the key drug in TBM treatment, is poor, as is that of ethambutol and streptomycin [75-77]. Isoniazid and pyrazinamide penetrate more readily [78,79]. Sufficient activity of rifampicin is key to successful treatment, demonstrated by the fact that rifampicin resistance is associated with $100 \%$ mortality [72]. Levels of rifampicin in the CSF are approximately $10-20 \%$ of those in plasma, depending on the severity of blood-brain barrier disruption [80]. The antimycobacterial activity of rifampicin is exposure- and concentration-dependent [81]. A relatively modest increase in administered doses of rifampicin (from $10 \mathrm{mg} / \mathrm{kg}$ to $13 \mathrm{mg} / \mathrm{kg}$ ) resulted in a significant increase $(65 \%)$ in mean plasma area under the curve $0-24 \mathrm{~h}\left(\mathrm{AUC}_{0-24 \mathrm{~h}}\right)$ and $49 \%$ increase in plasma $\mathrm{C}_{\max }$ without a significant increase in the rate of adverse events $[82,83]$. Increasing the dose of rifampicin may result in higher mycobacteriocidal activity in the brain and possibly greater survival. There is a need to improve antituberculous regimens tailored to the CSF penetration of drugs and pharmacokinetics and pharmacodynamics. Fluoroquinolones are an attractive option for the treatment of TBM because of their demonstrable in vitro activity, tolerability, good bioavailability and ease of administration. A pharmacokinetic study in patients with TBM found levofloxacin to have excellent CSF penetration, with a ratio of AUC in CSF to AUC in plasma of $75 \%$. This compared favorably with gatifloxacin $(35 \%)$ and ciprofloxacin (14\%) [84].

A population pharmacokinetics study concluded that at comparative doses, levofloxacin showed the most favourable results. Median-free 
$\mathrm{AUC}_{0-24} /$ actual MIC $_{90}$ in plasma was 180.99 for levofloxacin, 179.77 for gatifloxacin and 58.35 for moxifloxacin [85].

Currently, a randomized trial is being conducted in Vietnam comparing a standard antitubercular treatment regimen with intensified treatment, in which patients receive a high dose of rifampicin $(15 \mathrm{mg} / \mathrm{kg} /$ day $)$ and additional levofloxacin $(20 \mathrm{mg} / \mathrm{kg})$ [58]. Improving the early sterilizing power of current therapy may result in improved outcome of all TBM patients.

The treatment regimen for patients with cerebral tuberculoma is generally identical to that used in TBM. Some studies advocate a longer duration of standard four-drug therapy [2]. Most lesions resolve with chemotherapy, but some require surgical intervention. HIV-positive patients with tuberculoma are more likely to experience seizures than HIV-negative patients [86]. Acute seizures occur in half of pediatric cases and in 5\% of adult cases of TBM [87]. Patients with seizures have a worse prognosis [6]. After the first acute seizure, TBM patients commonly experience recurrent seizures unless treated with antiepileptic drug prophylaxis; benzodiazepines in the acute phase, then phenytoin/fosphenytoin and subsequent maintenance therapy. Valproic acid should be avoided if possible due to hepatotoxicity [88].

\section{Drug-resistant TBM}

Drug resistance is a large and growing problem in many TB-endemic countries. Mutations can arise in Mtb that confer resistance to chemotherapy, and can be transmitted as easily as the original strain. Certain strains seem to be associated with increased drug resistance; for instance, the Beijing genotype is associated with fluoroquinolone and multidrug resistance in Vietnam [89]. MDR-TB is defined as Mtb with resistance to at least rifampicin and isoniazid.

In TBM, drug resistance may pose an even greater problem than in other forms of TB. Second-line TB drugs are less bactericidal and may have varying or unknown CSF penetration [90]. There are no uniform guidelines on how to treat MDR-TBM. DST results are often available after the patient has deteriorated or died. MDR-TBM is associated with up to $100 \%$ mortality $[45,72,90]$. Resistance to isoniazid only, or combined with streptomycin resistance, has not been associated with a significantly higher mortality in earlier reports [72]; however, in a larger study in HIV-positive patients, it was associated with increased mortality (adjusted HR: 1.78) (Figure 3) [45]. A retrospective cohort study showed a significant unadjusted association (odds ratio: 1.61) between isoniazid resistance and an increased risk of death in patients with positive CSF cultures [91]. Since isoniazid is the most effective bactericidal drug in the first 2 days of anti-TB treatment, early recognition of resistance is of great importance. Isolated isoniazid resistance or combined with resistance to other drugs was found in $32.3 \%$ of culture-positive samples in HIV patients with TBM, of which 13\% (overall MDR rate was 4.3\%) were MDR [45]. In a cohort with predominantly HIV-negative patients $(78.8 \%)$, isoniazid resistance was found in $37.1 \%$ of samples, of which $21 \%$ were MDR (overall MDR rate was 5.6\%) [72].

Clinical algorithms and treatment guidelines may need to be developed for clinicians to be able to make early treatment adjustments for those suspected of having drug-resistant TBM, before the availability of sensitivity tests. GeneXpert results showing rifampicin resistance may not be sufficient, as in some settings, MDR programs require a microbiological confirmation before patients are provided with secondline drugs. Diagnosis and treatment of drug resistance in TBM is a very significant problem, as demonstrated by the numbers published in recent reports.

\section{Immunomodulatory \& immune reconstituting treatment Thalidomide}

Thalidomide is a drug with immunomodulatory properties through the inhibition of TNF- $\alpha$. In animal studies, TNF- $\alpha$ levels in the CSF produced during TBM were shown to correlate with the extent of pathogenesis, although this has been difficult to replicate in humans with TBM [92]. The same group has used a thalidomide ana$\log$ (IMiD3) for the treatment of experimental animals infected with TBM as an adjunctive to standard antituberculous treatment. IMiD3 has comparable immunomodulatory action to thalidomide, but unlike thalidomide, IMiD3 is not teratogenic. The additional use of IMiD3 in rabbits resulted in marked improvement in survival, reduced CSF leukocytosis, lower levels of TNF, and attenuated inflammation of the meninges on histological examination. The effect on survival and severity of symptoms of IMiD3 was markedly greater than that of thalidomide in this animal model [93].

A randomized controlled study of adjunctive thalidomide for pediatric patients with TBM was stopped early because all deaths and adverse events occurred in the treatment arm. However, 
Survival by resistance category

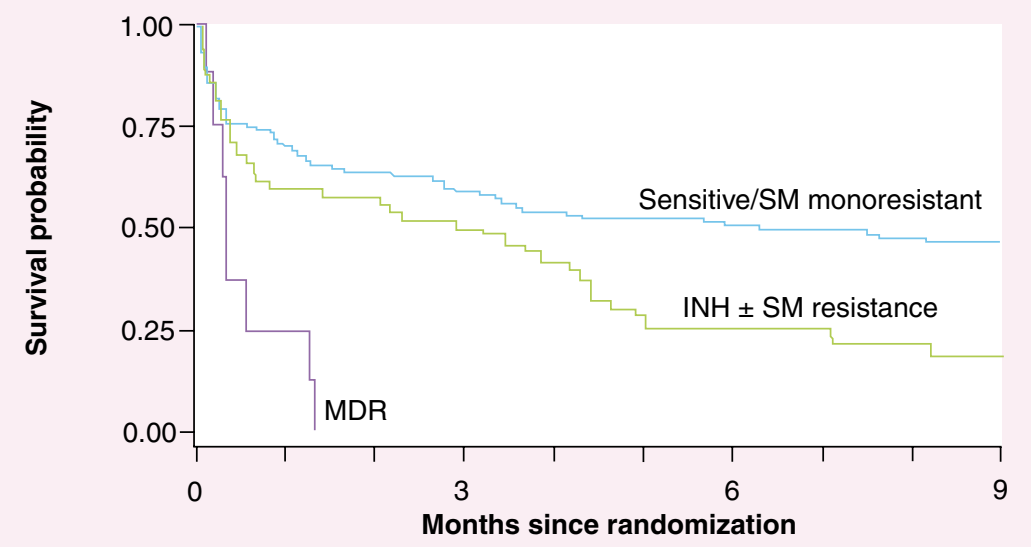

\begin{tabular}{|lllll|}
\hline Number at risk & & & & \\
Sensitive & 126 & 66 & 53 & 47 \\
INH \pm SM resistance & 52 & 25 & 11 & 8 \\
MDR & 8 & 0 & 0 & 0 \\
\hline
\end{tabular}

Figure 3. Survival by drug-resistance pattern of pathogen of a HIV-positive cohort. INH: Isoniazid; MDR: Multidrug resistant; SM: Streptomycin.

Reproduced with permision from [45].

the patients who died had very severe neurological symptoms at baseline [94]. The experimental evidence base for use of TNF- $\alpha$ inhibitors has been established, but the reported adverse events with thalidomide have deterred further research. Potentially, IMiD3 will play a role in future clinical research, as it has been shown to be less toxic and more effective in animal studies.

\section{Dexamethasone}

The rationale for using dexamethasone in TBM has been discussed in earlier sections. The mechanism of action of steroids is multiple; they may restore damaged vascular permeability, decrease CSF production, free-radical production and damage and are anti-inflammatory and immunomodulating. Addition of dexamethasone to the antibiotic regimen used in TBM has been proven to reduce mortality in TBM. Significantly fewer adverse effects were observed in the dexamethasone group as compared with the placebo group; however, it was not associated with a significant reduction in the proportion of severely disabled patients. In this study, the mortality reduction for HIV-positive patients did not reach statistical significance $(p=0.08)$ [4]. According to a recent Cochrane review, corticosteroids should be routinely used in HIV-negative people with TBM; however, current evidence does not support or refute a similar conclusion for HIV-positive patients [95].
Current global guidelines have included corticosterioids as an integral part of TBM treatment. The very recent genetic data, as described earlier [37], may warrant research in the direction of a more tailored use of corticosteroids according to individual severity of the immune response.

\section{Immune reconstitution with antiretroviral therapy}

The mortality for HIV-infected patients with TBM is alarmingly high [5,96,97]. Most patients presenting with TBM are either not receiving antiretrovirals (ARVs) or are not being effectively treated with them. CD4 counts of patients with TBM are typically very low (<50) [97]. For these patients, the question of timing of ART is critical. Initiation should be as early as possible to support the immune response; however, toxicity, the immune reconstitution syndrome (IRIS) and pill burden may complicate early treatment. Paradoxical TB-IRIS in TB/HIVpatients presents as worsening of symptoms after initial TB treatment response following initiation of ART [98]. Two recent clinical trials on initiation of ART in HIV-associated TB showed that severely immunocompromised (CD4<200) patients with TB may benefit from early initiation of ART, with lower rates of AIDS-defining illnesses or death, but at the cost of a higher occurrence of IRIS [99,100]. An editorial on these publications added that the majority of the patients had pulmonary TB, which has a 
reasonably good prognosis. IRIS is rarely lifethreatening for these patients. In TBM, mortality is much higher and intracranial IRIS may be detrimental [101]. The incidence of IRIS in TBM is unknown, diagnosis is difficult to establish, and it must be distinguished from other causes of neurological deterioration, such as drug resistance, other CNS opportunistic infections and stroke. Using a recently published case definition may improve recognition of this syndrome [98]. A recent randomized controlled trial showed no reduction in mortality with immediate initiation of ART; instead, the study suggests that it may be safer to defer the initiation of ART to 8 weeks of TBM treatment in patients with TBM, as fewer adverse events occurred in the delayed treatment group [5].

\section{Managing secondary effects Stroke}

Stroke is associated with poor outcome in TBM. It may occur in up to $60 \%$ of patients. Exudates may cause infarction through vasculitis. There is some evidence that strokes early in the course of disease are caused by vasospasms and later strokes involve proliferative intimal disease [17]. Antituberculous treatment does not appear to prevent stroke [17]. The use of aspirin has been researched in TBM, since it is antithrombotic and possibly neuroprotective. Children with TBM and adults with pulmonary TB have been shown to have hypercoagulation, which may contribute to the development of ischemia [102]. However, a study performed in children with TBM showed no significant effect on either mortality or neurological deficits with either low- or high-dose aspirin regimens [103]. A recent openlabel randomized study in adults on the role of aspirin showed a beneficial effect on mortality and MRI results [104]. However, in this study, patients selectively received corticosteroids, which may have biased the results. The role of aspirin in management of adult TBM remains to be established.

In a South African Neurosurgery Hospital, the cerebral tissue oxygenation (Ptio2) of two children with severe TBM was monitored. In one of the patients, a precipitous decline of Ptio2 was successfully reversed by aggressive therapy to treat cerebral hypoxia, along with fluid resuscitation, inotropic support and blood transfusion. The authors suggest that these interventions may have prevented infarction [105]. Even though it is unlikely these high-level monitoring techniques will become practice in low-resource settings, these are critical observations.
More profound insights into the pathogenesis of TBM vascular disease are necessary to guide rational therapeutic interventions.

\section{Raised intracranial pressure \\ \& hydrocephalus}

Management of intracranial pressure (ICP) and hydrocephalus is a neglected area in the treatment of TBM. There is no standard intervention strategy or evidence on which to base practice for raised ICP. Raised ICP and hydrocephalus in TBM are associated with a worse outcome. The most frequent cause of raised ICP is hydrocephalus. Hydrocephalus may be seen on the admission CT brain scan in adult patients $[19,106]$. Cerebral edema is a less common cause. If the ICP can no longer be compensated in the confines of the skull, it may result in brain herniation or reduced cerebral perfusion leading to ischemia.

Patients with hydrocephalus may present with nonspecific symptoms. If consciousness is reduced, hydrocephalus should be suspected, even if papilledema is absent. CT and MRI may provide information about the ventricular size and cause of hydrocephalus (tuberculoma, infarcts, basal exudate), but are insufficient to distinguish between communicating and noncommunicating hydrocephalus. In communicating hydrocephalus, CSF reabsorption is obstructed in the basal cisterns; in noncommunicating hydrocephalus, there is outlet blockage at the level of the fourth ventricle or the Aqueduct of Sylvius. Distinction between the two is important in terms of potential treatment options. Communicating hydrocephalus is more common in TBM (82\%) [107].

Communicating hydrocephalus can be treated with furosemide with or without acetazolamide. Some institutions favor daily lumbar punctures, with ICP monitoring through manometry [108].

Treatment of noncommunicating hydrocephalus is more challenging; lumbar puncture performed in those patients may result in coning and death. Treatment involves invasive neurosurgical procedures. An external ventricular drain, ventriculoperitoneal shunting or endoscopic third ventriculostomy may be indicated [108]. Patients for these procedures should be carefully selected, as the success rate depends on the correct diagnosis, the severity of disease and the expertise of the neurosurgical teams. Outcome is better in patients with early intervention in the better grades [109].

\section{Hyponatremia}

TBM, similar to other neurological disorders, is often accompanied by hyponatremia, which is 
associated with a worse outcome. Mostly implicated as a cause are the syndrome of inappropriate $\mathrm{ADH}$ secretion (SIADH) and cerebral salt wasting syndrome (CSW). It is critical to manage hyponatremic in patients appropriately, as a hypo-osmolar state will worsen brain edema. Both syndromes require a different approach, but it may be difficult to distinguish between the two. In SIADH, hypothalamic dysfunction results in an inappropriate secretion of $\mathrm{ADH}$ leading to retention of fluid, low osmolality and low sodium. Patients are often euvolemic or hypervolemic. In CSW, it is hypothesized that anti-natriuretic peptide levels are increased, leading to sodium loss in urine, accompanied by water loss. For SIADH, fluid restriction is part of management, but this can be detrimental for patients with CWS, who are hypovolemic and may deteriorate rapidly. Treatment for CWS is hypertonic saline or fludrocortisone. Some experts suggest that SIADH in TBM is overdiagnosed and since the distinction may be difficult, they advise to treat cautiously all patients with hyponatremia with hypertonic saline with or without fludrocortisone $[108,110]$. Care should be taken in all patients not to correct the sodium water balance too rapidly as this may result in central pontine myelinolysis. Optimization of supportive management of all accompanying complications may lead to an improvement in clinical outcome.

\section{Conclusion}

Tuberculous meningitis, a debilitating and in many cases lethal form of $\mathrm{TB}$, is associated with multiple challenges for both clinicians and researchers. The mortality remains high despite the introduction of anti-TB treatment; approximately $25 \%$ for HIV-negative and $67 \%$ for HIV-positive patients, with most deaths occurring within 1 month of presenting to hospital. The major prognostic factors influencing survival and disability include the length of delay of treatment initiation, TBM severity grade upon admission, the occurrence of stroke, seizures, hyponatremia, cranial nerve involvement, CSF cell count and lactate level, HIV coinfection and multidrug resistance.

Advances made in the last 5-10 years have altered both paradigms and practices in TBM. Based on pathological, post-mortem and animal studies, researchers have proposed a new paradigm of TBM pathogenesis focusing on the critical role of miliary $\mathrm{TB}$, challenging the view (dating back to 1933) present in the existing literature. In addition to the traditional microbiological and clinical diagnostic tools (i.e., smear and culture microscopy), recent advances have been made in the form of diagnostic tests: MODS and molecular techniques (i.e., GeneXpert). Although far from perfect, these tools bring us closer to successful management through early diagnosis of TBM. Studies on pathogen genetics have yielded the complete sequence of the Mtb pathogen and shown that some strains (Beijing) are potentially more pathogenic than others. Fewer studies have been performed on host genetics, and have generally focused on the role of the TLR family, which is believed to influence the dissemination of Mtb. Very recently, the $L T A 4 H$ genotype has been shown to play a role in the severity of the inflammatory response. This response may not be uniform in all patients. Both hypo- and hyper-inflammation may have a negative effect on the course of disease, warranting a review of the use of immunomodulatory treatment for all patients. Genetic analyses of both host and pathogen reveal that TBM progression and clinical phenotype may be multifactorial and involve interactions between bacterial and host genotypes.

Treatment for TBM is still largely based on pulmonary regimens, and treatment guidelines for TBM are not uniform or based on sound pharmacological principles. Treatment involves three components: the anti-TB drug regimen, management of secondary effects such as stroke and hydrocephalus, and immunomodulatory treatments (i.e., ARVs, thalidomide and dexamethasone). In HIV-positive patients, there is no benefit in early initiation of ARVs, and it may be safer to defer the initiation to 8 weeks of TBM treatment. Although thalidomide led to adverse effects in clinical trials in children, more research is needed on the less-toxic thalidomide analog IMiD3 in adults. Dexamethasone has been proven to reduce early mortality and adverse events, although not disability, in HIV-negative TBM patients. Drug resistance, and particularly MDR-TBM, is significantly associated with worse outcome. Despite decades of investment in drug discovery, no new antibiotics have been adopted in the last 40 years, and those that exist for pulmonary TB have highly variable levels of penetration into the CSF.

Recent research is pointing the way towards newer models that may lead to practical improvements in clinical care. Further clinical research should aim at the development and assessment of better diagnostics (especially for MDR-TBM), 
improving the specific antibiotic regimen for TBM, assessment of the role of neurosurgical intervention and studies aimed at a better understanding of the immunopathogenesis and potential adjunct immunomodulatory drugs, including potentially aspirin and thalidomide analog IMiD3. The development of standardized definitions to aid the design and analysis of clinical trials in TBM is a major step in the right direction.

\section{Future perspective}

New approaches to the diagnosis and treatment of TBM are urgently needed. Promising research is underway with the advent of new technologies such as the GeneXpert molecular diagnostic test, yet this has not been evaluated sufficiently for extrapulmonary $\mathrm{TB}$, nor has its cost-effectiveness been determined in resource-limited countries where TB incidence is high. Improved diagnostics are needed for a range of purposes, including acute infection, latent infection, drug resistance and contact-tracing.

Models show that the WHO goal of TB eradication by 2050 will be unreachable without an effective vaccine [111]. The only available vaccine, BCG, was developed in 1921 and provides limited and unreliable protection against TB. New vaccines are in the pipeline, but estimates vary widely regarding when they will become available for clinical use. In the last decade, 12 vaccines entered clinical trials; two are therapeutic vaccines for immune therapy in adjunct to ARV treatment for HIV-TB coinfected individuals, and of the other ten preventative vaccines are all either recombinant BCG constructs or boosters of BCG-prime vaccinations [112].

With regard to treatment, novel pharmaceuticals should be developed featuring lower toxicity, shorter and simpler treatment, decreased chance of resistance and higher CSF penetration; also needed are more imaginative ways of delivering these treatments. Rifampicin, discovered in 1963, is the last new drug to be adopted. As of 2010, ten drugs were in clinical trials - four existing drugs that were redeveloped for TB and six new chemical compounds [113] In addition, there were 34 compounds or projects in the discovery and preclinical stages; this highlights the need for novel approaches

\section{Executive summary}

\section{Burden of disease}

- Tuberculous meningitis (TBM) is difficult to diagnose and treat for a number of reasons: the pathology is poorly understood; rapid, sensitive and affordable diagnostics have yet to be developed; drug resistance is clearly crucial to the clinical outcome; and the optimal dose, duration and composition of anti-TB treatment regimen have still not been determined.

- The HIV pandemic has further complicated the management of TB, and infection is more likely to progress to meningeal TB when patients are coinfected with HIV. In HIV-negative adults, the mortality is approximately 25\%; for HIV-positive patients, it is $67 \%$. Approximately half of survivors suffer from long-term neurological sequelae.

\section{Immunopathogenesis}

- TBM morbidity and mortality are considered to be dependent on the severity of the inflammatory response and provided the rationale for using adjunctive treatment dexamethasone in some TBM patients. Immunological studies provide conflicting results, however, creating a paradox between the hypotheses and the evidence, further complicated by the association of TBM with HIV.

\section{Pathogen \& host genetics}

- Genetic analyses of both host and pathogen reveal that TBM progression and clinical phenotype may be multifactorial and involve interactions between bacterial and host genotypes.

- The Beijing genotype was significantly associated with HIV status, single- and multi-drug resistance, shorter duration of illness before presentation to hospital, and fewer CSF leukocytes - this suggests that mycobacterial genotype may affect disease progression and the nature of the intracerebral inflammatory response.

\section{Diagnosis}

- Diagnostic tests for TBM can be divided into different groups: biochemical tests that can detect features of the infecting organism or products of the host immune response; detection of mycobacterial antigens or antimycobacterial antibodies; and molecular tests that detect DNA fragments of mycobacteria.

- For TBM, rapid molecular tests may be of great importance, as delay in diagnosis is so directly linked to mortality.

\section{Treatment}

- Despite decades of investment in drug discovery, no new antibiotics have been adopted in the last 30 years.

- It is safer to defer the initiation of antiretroviral therapy to 8 weeks of TBM treatment rather than at the start in patients with TBM.

- Dexamethasone has been proven to reduce mortality and adverse events, though not disability, in HIV-negative TBM patients.

- There is a need to improve antituberculous regimens tailored to the CSF penetration of drugs and key pharmacokinetic and pharmacodynamic principles. 
for evaluating optimal drug combinations [114]. The crucial parameter in terms of significance for TBM control is drug CSF penetrance. Further investigation into basic science - pathways in mycobacterial cell wall biosynthesis, disease progression and host-pathogen genetic interactions - may lead to new approaches to treatment.

Major hurdles are not only scientific in nature; institutional and cultural roadblocks exist despite international consensus on the importance of TB control. Private industry is not fully engaged in research on what are seen as 'diseases of poverty', though public-private partnerships such as the Global Alliance for TB Drug Development are a step in the right direction. National governments like stability in health policy and practice, but in an era of biomedical advances, this reticence can hamper effective disease control. Many countries in which TB rates are high have strategies to deal with acute diseases, but less so with chronic infections. While vertical healthcare delivery systems are less expensive to run than multiple horizontal systems, they often exclude vulnerable populations where transmission continues. Within many communities, there is stigma associated with HIV, which is often comorbid with TB; these social barriers complicate the path towards treatment and should not be ignored when structuring new interventions. Biomedical research is there to provide evidence and push boundaries; yet meaningful collaboration between researchers, clinicians, policymakers and international agencies is needed to ensure that discoveries are incorporated into policy and practice as soon as possible.

\section{Financial \& competing interests disclosure \\ The authors have no relevant affliations or financial involvement with any organization or entity with a financial interest in or financial conflict with the sub- ject matter or materials discussed in the manuscript. This includes employment, consultancies, honoraria, stock ownership or options, expert testimony, grants or patents received or pending, or royalties. \\ No writing assistance was utilized in the production of this manuscript.}

\section{References}

1. WHO Global Tuberculosis Programme. Treatment of Tuberculosis: Guidelines for National Programmes. WHO Press, Switzerland, 108 (2010).

2. Chatterjee S. Brain tuberculomas, tubercular meningitis, and post-tubercular hydrocephalus in children. J. Pediatr. Neurosci. 6(Suppl. 1), S96-S100 (2011).

3. Thwaites GE, Tran TH. Tuberculous meningitis: many questions, too few answers. Lancet Neurol. 4(3), 160-170 (2005).

4. Thwaites GE, Nguyen DB, Nguyen HD et al. Dexamethasone for the treatment of tuberculous meningitis in adolescents and adults. N. Engl. J. Med. 351(17), 1741-1751 (2004).

5. Torok ME, Yen NT, Chau TT et al. Timing of initiation of antiretroviral therapy in human immunodeficiency virus (HIV)associated tuberculous meningitis. Clin. Infect. Dis. 52(11), 1374-1383 (2011).

6. Hosoglu S, Geyik MF, Balik I et al. Predictors of outcome in patients with tuberculous meningitis. Int. J. Tuberc. Lung Dis. 6(1), 64-70 (2002).

7. Kalita J, Misra UK, Ranjan P. Predictors of long-term neurological sequelae of tuberculous meningitis: a multivariate analysis. Eur. J. Neurol. 14(1), 33-37 (2007).

8. Thwaites GE, Simmons CP, Than Ha Quyen $\mathrm{N}$ et al. Pathophysiology and prognosis in vietnamese adults with tuberculous meningitis. J. Infect. Dis. 188(8), 1105-1115 (2003).

9. Torok ME, Nguyen DB, Tran TH et al. Dexamethasone and long-term outcome of tuberculous meningitis in Vietnamese adults and adolescents. PLoS One 6(12), e27821 (2011).

10. Medical Research Council. Streptomycin treatment of tuberculous meningitis. $B M J$ 582-597 (1948).

11. Donald PR, Schaaf HS, Schoeman JF. Tuberculous meningitis and miliary tuberculosis: the Rich focus revisited. J. Infect. 50(3), 193-195 (2005).

12. Rich AR, McCordock HA. The pathogenesis of tuberculous meningitis. Bull. John Hopkins Hosp. 52, 2-37 (1933).

13. Katti MK. Pathogenesis, diagnosis, treatment, and outcome aspects of cerebral tuberculosis. Med. Sci. Monit. 10(9), RA215-229 (2004).

14. Thwaites G, Chau TT, Mai NT, Drobniewski F, Mcadam K, Farrar J. Tuberculous meningitis. J. Neurol. Neurosurg. Psych. 68(3), 289-299 (2000).

15. North RJ, Jung YJ. Immunity to tuberculosis. Ann. Rev. Immunol. 22, 599-623 (2004).

16. Donald PR, Schoeman JF. Tuberculous meningitis. N. Engl. J. Med. 351(17), 1719-1720 (2004).

17. Lammie GA, Hewlett RH, Schoeman JF, Donald PR. Tuberculous cerebrovascular disease: a review. J. Infect. 59(3), 156-166 (2009).
18. Bernaerts A, Vanhoenacker FM, Parizel PM et al. Tuberculosis of the central nervous system: overview of neuroradiological findings. Eur. Radiol. 13(8), 1876-1890 (2003).

19. Thwaites GE, Macmullen-Price J, Tran TH et al. Serial MRI to determine the effect of dexamethasone on the cerebral pathology of tuberculous meningitis: an observational study. Lancet Neurol. 6(3), 230-236 (2007).

20. Wasay M, Kheleani BA, Moolani MK et al. Brain CT and MRI findings in 100 consecutive patients with intracranial tuberculoma. J. Neuroimaging 13(3), 240-247 (2003).

21. Wasay M, Moolani MK, Zaheer J, Kheleani BA, Smego RA, Sarwari RA. Prognostic indicators in patients with intracranial tuberculoma: a review of 102 cases. J. Pak. Med. Assoc. 54(2), 83-87 (2004).

22. Roos KL. Mycobacterium tuberculosis meningitis and other etiologies of the aseptic meningitis syndrome. Semin. Neurol. 20(3), 329-335 (2000).

23. Anuradha HK, Garg RK, Sinha MK et al. Intracranial tuberculomas in patients with tuberculous meningitis: predictors and prognostic significance. Int. J. Tuberc. Lung Dis. 15(2), 234-239 (2011).

24. Sharma P, Garg RK, Verma R, Singh MK, Shukla R. Incidence, predictors and prognostic value of cranial nerve involvement in patients with tuberculous meningitis: a retrospective evaluation. Eur. J. Intern. Med. 22(3), 289-295 (2011). 
25. Villoria MF, Fortea F, Moreno S, Munoz L, Manero M, Benito C. MR imaging and CT of central nervous system tuberculosis in the patient with AIDS. Radiol. Clin. North Am. 33(4), 805-820 (1995).

26. Kalita J, Prasad S, Maurya PK, Kumar S, Misra UK. MR angiography in tuberculous meningitis. Acta Radiol. 53(3), 324-329 (2012).

27. Nair PP, Kalita J, Kumar S, Misra UK. MRI pattern of infarcts in basal ganglia region in patients with tuberculous meningitis. Neuroradiology 51(4), 221-225 (2009).

28. Shukla R, Abbas A, Kumar P, Gupta RK, Jha $S$, Prasad KN. Evaluation of cerebral infarction in tuberculous meningitis by diffusion weighted imaging. J. Infect. 57(4), 298-306 (2008).

29. Misra UK, Kalita J, Maurya PK. Stroke in tuberculous meningitis. J. Neurol. Sci. 303(1-2), 22-30 (2011).

30. Kalita J, Misra UK, Nair PP. Predictors of stroke and its significance in the outcome of tuberculous meningitis. J. Stroke Cerebrovasc. Dis. 18(4), 251-258 (2009).

31. Simmons CP, Thwaites GE, Quyen NT et al. Pretreatment intracerebral and peripheral blood immune responses in Vietnamese adults with tuberculous meningitis: diagnostic value and relationship to disease severity and outcome. J. Immunol. 176(3), 2007-2014 (2006).

32. Green JA, Thi Hong Chau T, Farrar JJ, Friedland JS, Thwaites GE. CNS infection, CSF matrix metalloproteinase concentrations, and clinical/laboratory features. Neurology 76(6), 577-579 (2011).

33. Green JA, Tran CT, Farrar JJ et al. Dexamethasone, cerebrospinal fluid matrix metalloproteinase concentrations and clinical outcomes in tuberculous meningitis. PLoS One 4(9), e7277 (2009).

34. Cecchini D, Ambrosioni J, Brezzo C et al. Tuberculous meningitis in HIV-infected and non-infected patients: comparison of cerebrospinal fluid findings. Int. J. Tuberc. Lung Dis. 13(2), 269-271 (2009).

35. Rock RB, Hu S, Gekker G et al. Mycobacterium tuberculosis-induced cytokine and chemokine expression by human microglia and astrocytes: effects of dexamethasone. J. Infect. Dis. 192(12), 2054-2058 (2005).

36. Simmons CP, Thwaites GE, Quyen NT et al. The clinical benefit of adjunctive dexamethasone in tuberculous meningitis is not associated with measurable attenuation of peripheral or local immune responses. J. Immunol. 175(1), 579-590 (2005).

37. Tobin DM, Roca FJ, Oh SF et al. Host genotype-specific therapies can optimize the inflammatory response to mycobacterial infections. Cell 148(3), 434-446 (2012).

38. Trunz BB, Fine P, Dye C. Effect of BCG vaccination on childhood tuberculous meningitis and miliary tuberculosis worldwide: a meta-analysis and assessment of costeffectiveness. Lancet 367(9517), 1173-1180 (2006).

39. Rodrigues LC, Mangtani P, Abubakar I. How does the level of BCG vaccine protection against tuberculosis fall over time? BMJ 343 , d5974 (2011).

40. Sreevatsan S, Pan X, Stockbauer KE et al. Restricted structural gene polymorphism in the Mycobacterium tuberculosis complex indicates evolutionarily recent global dissemination. Proc. Natl Acad. Sci. USA 94(18), 9869-9874 (1997).

41. Glynn JR, Alghamdi S, Mallard K et al. Changes in Mycobacterium tuberculosis genotype families over 20 years in a population-based study in northern Malawi. PLoS One 5(8), e12259 (2010).

42. Caws M, Thwaites G, Stepniewska K et al. Beijing genotype of Mycobacterium tuberculosis is significantly associated with human immunodeficiency virus infection and multidrug resistance in cases of tuberculous meningitis. J. Clin. Microbiol. 44(11), 3934-3939 (2006).

43. Thwaites G, Caws M, Chau TT et al. Relationship between Mycobacterium tuberculosis genotype and the clinical phenotype of pulmonary and meningeal tuberculosis. J. Clin. Microbiol. 46(4), 1363-1368 (2008).

44. Faksri K, Drobniewski F, Nikolayevskyy V et al. Epidemiological trends and clinical comparisons of Mycobacterium tuberculosis lineages in Thai TB meningitis. Tuberculosis (Edinb.) 91(6), 594-600 (2011).

45. Tho DQ, Torok ME, Yen NT et al. Influence of antituberculosis drug resistance and Mycobacterium tuberculosis lineage on outcome in HIV-associated tuberculous meningitis. Antimicrob. Agents Chemother. 56(6), 3074-3079 (2012).

46. Awomoyi AA, Marchant A, Howson JM, Mcadam KP, Blackwell JM, Newport MJ. Interleukin-10, polymorphism in SLC11A1 (formerly NRAMP1), and susceptibility to tuberculosis. J. Infect. Dis. 186(12), 1808-1814 (2002).

47. Bellamy R. Identifying genetic susceptibility factors for tuberculosis in Africans: a combined approach using a candidate gene study and a genome-wide screen. Clin. Sci. (Lond.) 98(3), 245-250 (2000).

48. Bellamy R. Susceptibility to mycobacterial infections: the importance of host genetics. Genes Immun. 4(1), 4-11 (2003).
49. Spira A, Carroll JD, Liu G et al. Apoptosis genes in human alveolar macrophages infected with virulent or attenuated Mycobacterium tuberculosis: a pivotal role for tumor necrosis factor. Am. J. Respir. Cell Mol. Biol. 29(5), 545-551 (2003).

50. Kleinnijenhuis J, Oosting M, Joosten LA, Netea MG, Van Crevel R. Innate immune recognition of Mycobacterium tuberculosis. Clin. Develop. Immunol. 2011, 405310 (2011).

51. Thuong NT, Dunstan SJ, Chau TT et al. Identification of tuberculosis susceptibility genes with human macrophage gene expression profiles. PLoS Pathog. 4(12), e1000229 (2008).

52. Hawn TR, Dunstan SJ, Thwaites GE et al. A polymorphism in Toll-interleukin 1 receptor domain containing adaptor protein is associated with susceptibility to meningeal tuberculosis. J. Infect. Dis. 194(8), 1127-1134 (2006).

53. Thwaites GE, Chau TT, Farrar JJ. Improving the bacteriological diagnosis of tuberculous meningitis. J. Clin. Microbiol. 42(1), 378-379 (2004).

54. Kumar P, Srivatsava MV, Singh S, Prasad HK. Filtration of cerebrospinal fluid improves isolation of mycobacteria. J. Clin. Microbiol. 46(8), 2824-2825 (2008).

55. Caws M, Dang TM, Torok E et al. Evaluation of the MODS culture technique for the diagnosis of tuberculous meningitis. PLoS One 2(11), e1173 (2007).

56. Ha DT, Lan NT, Wolbers M et al. Microscopic observation drug susceptibility assay (MODS) for early diagnosis of tuberculosis in children. PLoS One 4(12), e8341 (2009).

57. Hanif SN, Eldeen HS, Ahmad S, Mokaddas E. GeneXpert(R) MTB/RIF for rapid detection of Mycobacterium tuberculosis in pulmonary and extra-pulmonary samples. Int. J. Tuberc. Lung Dis. 15(9), 1274-1275 (2011).

58. Heemskerk D, Day J, Chau TT et al. Intensified treatment with high dose rifampicin and levofloxacin compared to standard treatment for adult patients with tuberculous meningitis (TBM-IT): protocol for a randomized controlled trial. Trials 12 , 25 (2011).

59. Marais S, Thwaites G, Schoeman JF et al. Tuberculous meningitis: a uniform case definition for use in clinical research. Lancet Infect. Dis. 10(11), 803-812 (2010).

60. Brady T. Adenosine deaminase. Biochem. J. 36(5-6), 478-484 (1942).

61. Ocana I, Martinez-Vazquez JM, Segura RM, Fernandez-De-Sevilla T, Capdevila JA. Adenosine deaminase in pleural fluids. Test for diagnosis of tuberculous pleural effusion. Chest 84(1), 51-53 (1983). 
62. Xu HB, Jiang RH, Li L, Sha W, Xiao HP. Diagnostic value of adenosine deaminase in cerebrospinal fluid for tuberculous meningitis: a meta-analysis. Int. J. Tuberc. Lung Dis. 14(11), 1382-1387 (2010).

63. Garcia-Monco C, Berciano J. Sarcoid meningitis, high adenosine deaminase levels in CSF and results of cranial irradiation. J. Neurol. Neurosurg. Psych. 51(12), 1594-1596 (1988).

64. Lopez-Cortes LF, Cruz-Ruiz M, GomezMateos J et al. Adenosine deaminase activity in the CSF of patients with aseptic meningitis: utility in the diagnosis of tuberculous meningitis or neurobrucellosis. Clin. Infect. Dis. 20 (3), 525-530 (1995).

65. Chan J, Fan XD, Hunter SW, Brennan PJ, Bloom BR. Lipoarabinomannan, a possible virulence factor involved in persistence of Mycobacterium tuberculosis within macrophages. Infect. Immun. 59(5), 1755-1761 (1991).

66. Patel VB, Singh R, Connolly C et al. Comparison of a clinical prediction rule and a LAM antigen-detection assay for the rapid diagnosis of TBM in a high HIV prevalence setting. PLoS One 5(12), e15664 (2010).

67. Pai M, Flores LL, Pai N, Hubbard A, Riley LW, Colford JM Jr. Diagnostic accuracy of nucleic acid amplification tests for tuberculous meningitis: a systematic review and meta-analysis. Lancet Infect. Dis. 3(10), 633-643 (2003).

68. Pai M, Ling DI. Rapid diagnosis of extrapulmonary tuberculosis using nucleic acid amplification tests: what is the evidence? Future Microbiol. 3(1), 1-4 (2008).

69. Evans CA. GeneXpert - a game-changer for tuberculosis control? PLoS Med. 8(7), e1001064 (2011).

70. Boehme CC, Nicol MP, Nabeta P et al. Feasibility, diagnostic accuracy, and effectiveness of decentralised use of the Xpert MTB/RIF test for diagnosis of tuberculosis and multidrug resistance: a multicentre implementation study. Lancet 377(9776), 1495-1505 (2011).

71. Hillemann D, Rusch-Gerdes S, Boehme C, Richter E. Rapid molecular detection of extrapulmonary tuberculosis by the automated GeneXpert MTB/RIF system. J. Clin. Microbiol. 49(4), 1202-1205 (2011).

72. Thwaites GE, Lan NT, Dung NH et al. Effect of antituberculosis drug resistance on response to treatment and outcome in adults with tuberculous meningitis. J. Infect. Dis. 192(1), 79-88 (2005).

73. Thwaites G, Fisher M, Hemingway C, Scott $\mathrm{G}$, Solomon T, Innes J. British Infection Society guidelines for the diagnosis and treatment of tuberculosis of the central nervous system in adults and children. J. Infect. 59(3), 167-187 (2009).

74. American Thoracic Society; CDC; Infectious Diseases Society of America. Treatment of tuberculosis. MMWR Recomm. Rep. 52(RR-11), 1-77 (2003).

75. McIlleron H, Wash P, Burger A, Norman J, Folb PI, Smith P. Determinants of rifampin, isoniazid, pyrazinamide, and ethambutol pharmacokinetics in a cohort of tuberculosis patients. Antimicrob. Agents Chemother. 50(4), 1170-1177 (2006).

76. Tappero JW, Bradford WZ, Agerton TB et al. Serum concentrations of antimycobacterial drugs in patients with pulmonary tuberculosis in Botswana. Clin. Infect. Dis. 41(4), 461-469 (2005).

77. Wilkins JJ, Langdon G, McIlleron H, Pillai GC, Smith PJ, Simonsson US. Variability in the population pharmacokinetics of pyrazinamide in South African tuberculosis patients. Eur. J. Clin. Pharmacol. 62(9), 727-735 (2006).

78. Ellard GA, Humphries MJ, Gabriel M, Teoh R. Penetration of pyrazinamide into the cerebrospinal fluid in tuberculous meningitis. Br. Med. J. (Clin. Res. Ed.) 294(6567), 284-285 (1987).

79. Barling RW, Selkon JB. The penetration of antibiotics into cerebrospinal fluid and brain tissue. J. Antimicrob. Chemother. 4(3), 203-227 (1978).

80. Krishnan N, Malaga W, Constant $\mathrm{P}$ et al. Mycobacterium tuberculosis lineage influences innate immune response and virulence and is associated with distinct cell envelope lipid profiles. PLoS One 6(9), e23870 (2011).

81. Gumbo T, Louie A, Deziel MR et al. Concentration-dependent Mycobacterium tuberculosis killing and prevention of resistance by rifampin. Antimicrob. Agents Chemother. 51(11), 3781-3788 (2007).

82. Ruslami R, Nijland H, Aarnoutse R et al. Evaluation of high-versus standard-dose rifampin in Indonesian patients with pulmonary tuberculosis. Antimicrob. Agents Chemother. 50(2), 822-823 (2006).

83. Ruslami R, Nijland HM, Alisjahbana B, Parwati I, Van Crevel R, Aarnoutse RE. Pharmacokinetics and tolerability of a higher rifampin dose versus the standard dose in pulmonary tuberculosis patients. Antimicrob. Agents Chemother. 51(7), 2546-2551 (2007).

84. Thwaites GE, Bhavnani SM, Chau TT et al. Randomized pharmacokinetic and pharmacodynamic comparison of fluoroquinolones for tuberculous meningitis.
Antimicrob. Agents Chemother. 55(7), 3244-3253 (2011).

85. Peloquin CA, Hadad DJ, Molino LP et al. Population pharmacokinetics of levofloxacin, gatifloxacin, and moxifloxacin in adults with pulmonary tuberculosis. Antimicrob. Agents Chemother. 52(3), 852-857 (2008).

86. Martinez-Vazquez C, Bordon J, RodriguezGonzalez A et al. Cerebral tuberculoma a comparative study in patients with and without HIV infection. Infection 23(3), 149-153 (1995).

87. Udani PM, Parekh UC, Dastur DK. Neurological and related syndromes in CNS tuberculosis. Clinical features and pathogenesis. J. Neurol. Sci. 14(3), 341-357 (1971).

88. Murthy JM. Tuberculous meningitis: the challenges. Neurol. India 58(5), 716-722 (2010).

89. Duong DA, Nguyen TH, Nguyen TN et al. Beijing genotype of Mycobacterium tuberculosis is significantly associated with high-level fluoroquinolone resistance in Vietnam. Antimicrob. Agents Chemother. 53(11), 4835-4839 (2009).

90. Patel VB, Padayatchi N, Bhigjee AI et al. Multidrug-resistant tuberculous meningitis in KwaZulu-Natal, South Africa. Clin Infect Dis 38(6), 851-856 (2004).

91. Vinnard C, Winston CA, Wileyto EP, Macgregor RR, Bisson GP. Isoniazid resistance and death in patients with tuberculous meningitis: retrospective cohort study. BMJ 341, c4451 (2010).

92. Tsenova L, Bergtold A, Freedman VH, Young RA, Kaplan G. Tumor necrosis factor alpha is a determinant of pathogenesis and disease progression in mycobacterial infection in the central nervous system. Proc. Natl Acad. Sci. USA 96(10), 5657-5662 (1999).

93. Tsenova L, Mangaliso B, Muller G et al. Use of IMiD3, a thalidomide analog, as an adjunct to therapy for experimental tuberculous meningitis. Antimicrob. Agents Chemother. 46(6), 1887-1895 (2002).

94. Schoeman JF, Springer P, Van Rensburg AJ et al. Adjunctive thalidomide therapy for childhood tuberculous meningitis: results of a randomized study. J. Child Neurol. 19(4), 250-257 (2004).

95. Prasad K, Singh MB. Corticosteroids for managing tuberculous meningitis. Cochrane Database Syst. Rev. (1), CD002244 (2008).

96. Thwaites GE, Duc Bang N, Huy Dung N et al. The influence of HIV infection on clinical presentation, response to treatment, and outcome in adults with tuberculous meningitis. J. Infect. Dis. 192(12), 2134-2141 (2005). 


\section{Review Brancusi, Farrar \& Heemskerk}

97. Torok ME, Chau TT, Mai PP et al. Clinical and microbiological features of HIVassociated tuberculous meningitis in Vietnamese adults. PLoS One 3(3), e1772 (2008).

98. Meintjes G, Lawn SD, Scano F et al. Tuberculosis-associated immune reconstitution inflammatory syndrome: case definitions for use in resource-limited settings. Lancet Infect. Dis. 8(8), 516-523 (2008).

99. Abdool Karim SS, Naidoo K, Grobler A et al. Integration of antiretroviral therapy with tuberculosis treatment. N. Engl. J. Med. 365(16), 1492-1501 (2011).

100. Blanc FX, Sok T, Laureillard D et al. Earlier versus later start of antiretroviral therapy in HIV-infected adults with tuberculosis. N. Engl. J. Med. 365(16), 1471-1481 (2011).

101. Torok ME, Farrar JJ. When to start antiretroviral therapy in HIV-associated tuberculosis. N. Engl. J. Med. 365(16), 1538-1540 (2011).

102. Schoeman J, Mansvelt E, Springer P, Van Rensburg AJ, Carlini S, Fourie E. Coagulant and fibrinolytic status in tuberculous meningitis. Pediatr. Infect. Dis. J. 26(5), 428-431 (2007).

103. Schoeman JF, Janse Van Rensburg A, Laubscher JA, Springer P. The role of aspirin in childhood tuberculous meningitis. J. Child Neurol. 26(8), 956-962 (2011).

104. Misra UK, Kalita J, Nair PP. Role of aspirin in tuberculous meningitis: a randomized open label placebo controlled trial. J. Neurol. Sci. 293(1-2), 12-17 (2010).

105. Figaji AA, Sandler SI, Fieggen AG, Le Roux PD, Peter JC, Argent AC. Continuous monitoring and intervention for cerebral ischemia in tuberculous meningitis. Pediatr. Crit. Care Med. 9(4), e25-30 (2008).

106. Ranjan P, Kalita J, Misra UK. Serial study of clinical and CT changes in tuberculous meningitis. Neuroradiology 45(5), 277-282 (2003).

107. Schoeman J, Donald P, Van Zyl L, Keet M, Wait J. Tuberculous hydrocephalus: comparison of different treatments with regard to ICP, ventricular size and clinical outcome. Develop. Med. Child Neurol. 33(5), 396-405 (1991).
108. Figaji AA, Fieggen AG. The neurosurgical and acute care management of tuberculous meningitis: Evidence and current practice. Tuberculosis (Edinb.) 90(6), 393-400 (2010).

109. Rajshekhar V. Management of hydrocephalus in patients with tuberculous meningitis. Neurol. India 57(4), 368-374 (2009).

110. Murthy JM. Management of intracranial pressure in tuberculous meningitis. Neurocrit. Care 2(3), 306-312 (2005).

111. Smith R. Eradication of tuberculosis by 2050 impossible without new vaccine. BMJ 338, b1291 (2009).

112. Kaufmann SH. Fact and fiction in tuberculosis vaccine research: 10 years later. Lancet Infect. Dis. 11(8), 633-640 (2011).

113. Ma Z, Lienhardt C, Mcilleron H, Nunn AJ, Wang X. Global tuberculosis drug development pipeline: the need and the reality. Lancet 375(9731), 2100-2109 (2010).

114. Lienhardt C, Vernon A, Raviglione MC. New drugs and new regimens for the treatment of tuberculosis: review of the drug development pipeline and implications for national programmes. Curr. Opin. Pulm. Med. 16(3), 186-193 (2010). 